\title{
Discusión final en torno a la formación y la investigación ${ }^{1}$ \\ https://doi.org/10.21830/9789585241459.11
}

Luz Adriana Albornoz Rodríguez Escuela de Armas Combinadas del Ejército

En este aparte se reúnen de manera general los principales aportes de las investigaciones y se exponen posibles horizontes investigativos que permitan profundizar en las temáticas abordadas. Esta mirada de síntesis reconoce el papel de los maestros que orientan los procesos educativos en las escuelas del Ejército, comprendiendo que ser maestro no es un quehacer estático ni único, sino que, por el contrario, es móvil y múltiple, como señala Álvarez (2018):

No hay una identidad por buscar en el pasado, ni por construir en el futuro; ser maestro ha sido algo muy diverso y el oficio ha cambiado radicalmente con el tiempo, en relación con sus fines políticos, con su importancia social, con el tipo de conocimiento y de valores que enseña. (p. 88)

Por esto, comprender los procesos de profesionalización del docente militar es importante a la luz de los nuevos paradigmas de la educación y las condiciones históricas que enfrenta el país y la fuerza.

En este contexto, un primer aspecto común en los trabajos fue el papel transformador de los docentes en los procesos de formación, gestión de las instituciones educativas, la investigación y el desarrollo de un saber pedagógico en contextos de formación militar. A partir de lo anterior, se afirma que la práctica pedagógica reposiciona al docente como un sujeto prudente, es decir, mantiene una "relación cons-

1 Capítulo de libro que parte de los resultados del proyecto de investigación Práctica Pedagógica en el Ejército Nacional: Reflexiones en torno a la Formación y la Investigación, del Grupo de Investigación para la Capacitación Militar (GICAM) de la Escuela de Armas Combinadas (ESACE), registrado con el código COL0160714 de Minciencias. Los puntos de vista y los resultados de este capítulo pertenecen a la autora y no reflejan necesariamente los de las instituciones participantes.

2 Licenciada en Educación Básica con Énfasis en Ciencias Sociales de la Universidad Pedagógica Nacional. Especialista en Entornos Virtuales de Aprendizaje del Centro de Altos Estudios Universitarios. Magíster en Desarrollo Educativo Social de la Fundación Centro Internacional de Educación y Desarrollo Humano. Candidata a doctora en Educación de la Universidad Pedagógica Nacional. Asesora de investigación de la Escuela de Armas Combinadas del Ejército (Esace). Orcid: https://orcid.org/0000-0002-9673-8776. Contacto: luzalbornozrodriguez@cedoc.edu.co 
titutiva con el conocimiento práctico, deliberación y la búsqueda del bien humano" (Carr, 2002, p. 100; citado por Barragán, 2012, p. 24) Además, apela a superar el quehacer como un conjunto de acciones ritualizadas en el que las instituciones a través de sus dispositivos de saber-poder imponen las lógicas del régimen de la verdad, con lo cual hace que parezcan singulares, únicos los procesos de evaluación y gestión educativa.

De ahí que la investigación sobre lo que acontece en las escuelas o centros de capacitación es necesaria, puesto que permite develar las buenas prácticas y orientar otras con el fin de generar conocimiento sobre los fenómenos estudiados. Además, favorece la deconstrucción, problematización y lectura de las instituciones desde diferentes perspectivas en las que se reconocen subjetividades (perspectivas de género) y prácticas discursivas que deben ser reorientadas para responder de manera más asertiva a los retos que esta sociedad impone.

Visto de esta manera, tanto los docentes como los estudiantes han desarrollado un conjunto de competencias, habilidades blandas y duras, que les han permitido reconocer, analizar y comprender los retos actuales, como lo ha enunciado Han (2012): "La sociedad del siglo XXI ya no es disciplinaria, sino una sociedad del rendimiento. Tampoco sus habitantes se llaman ya sujetos de obediencia, sino sujetos de rendimientos. Estos sujetos son emprendedores de sí mismos" (p. 25), lo cual genera lógicas de deshumanización que afectan el desarrollo humano.

Es claro que la sociedad actual se caracteriza por la aceleración, la inmediatez, la creación masiva y la complejidad del conocimiento. Esto exige que las personas desarrollen una mayor capacidad de administrar el tiempo, habilidades de literacidad crítica, informacionales y de trabajo en grupo, entre otras competencias de orden cognitivo y procedimental, puesto que deben acceder a diferentes fuentes de información, creación, producción, distribución y consumo. Es importante resaltar que la cooperación y colaboración contrarresta la individualidad, la competencia entre otras lógicas de la sociedad actual.

Puede señalarse entonces que gracias al conjunto de las diferentes habilidades, las personas pueden participar en las actividades que la cibercultura produce a través de los soportes análogos o digitales. Por eso, no es un asunto menor cuando se piensa en la importancia de las instituciones educadoras, pues ellas hacen posible el desarrollo de gran parte de las habilidades y competencias. Por esta razón las investigaciones de este libro hacen un llamado a complejizar nuestra visión del aprendizaje basado en competencias desde un enfoque socioformativo.

Desde esta perspectiva, la formación debe proveer los escenarios necesarios para desarrollar competencias específicas. Este es el caso de los oficiales y subofi- 
ciales de Logística, para quienes los investigadores han considerado 45 competencias que contribuyen a definir su perfil laboral y profesional. Se trata del resultado de un proceso riguroso de comparación y análisis con diferentes fuentes bibliográficas y perfiles de formación en el campo de las ciencias administrativas y contables.

Esta información es de gran importancia para la fuerza, toda vez que permite pensar en los procesos de certificación, homologación y ajuste de los programas. Además de identificar los elementos necesarios para la profesionalización, que según Cortina (2006) son: (1) contar con un carácter único, claramente definido socialmente; (2) ser considerado un servicio indispensable; (3) los que participan y se forman en ella tienen la convicción de su vocación; (4) forman una comunidad de profesionales-agremiaciones; (5) tienden a obtener un poder monopolístico; (6) las personas acceden a la profesión a través de un largo proceso de formación, y (7) existe un grado de autonomía en el ejercicio de su profesión, por lo cual (8) emite códigos deontológicos para su regulación.

Teniendo en cuenta lo anterior y a la luz de las reflexiones sobre las competencias, la figura 1 muestra los aspectos más relevantes tratados por los investigadores. Se incorpora a la gráfica las funciones de la conducción de la guerra como articulador de las diferentes habilidades que desarrollan los oficiales y suboficiales en el contexto de su hacer profesional.

\section{FUNCIONES DE CONDUCCIÓN DE LA GUERRA}

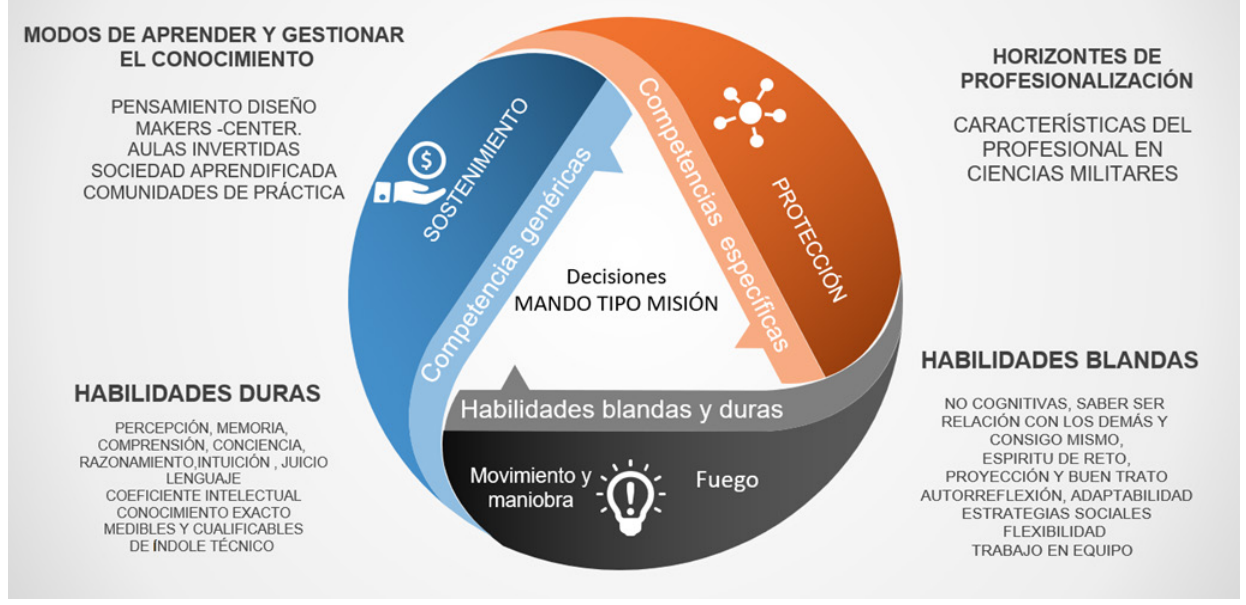

Figura 1. Aspectos relevantes de la formación en competencias y su relación con las habilidades duras y blandas.

Fuente: Original de la autora. 
En relación con lo anterior, el aprendizaje, la enseńanza y, de manera particular, el uso de estrategias asociadas a la didáctica disruptiva o a principios constructivistas fue un aspecto problematizado en las investigaciones, toda vez que se hizo alusión a la creación e innovación a partir de que cada uno haga las cosas (Do-It-Yourself, DIY) y en colaboración con otras personas (Do-It-With-Others, $D I W O)$, que incide en la manera de pensar las etapas o ciclos de formación y los modos convencionales en los que se comparte y transmite el conocimiento. Esto impacta en las prácticas de formación por cuanto los jóvenes que ingresan a la carrera militar acceden a diferentes plataformas y contenidos, independientemente de que en algunas oportunidades sean hábiles y expertos en el uso de la tecnología o cuenten con un desarrollo básico informacional.

Estas discusiones sobre la manera en que se gestiona la información son visibles en los nuevos paradigmas de la organización y gestión escolar, la investigación como producción nodal y la construcción de espacios para repensar la manera como se tramita el conflicto. Por ejemplo, los aportes sobre la gestión educativa hacen mayor énfasis en los procesos de descentralización, en los cuales la actividad de gestión transita de la gestión del sistema en su conjunto, a la gestión de un sistema que está compuesto por distintas entidades y niveles de competencia de gestión. Por eso se destaca el liderazgo pedagógico y no las miradas tecnocráticas de la gestión educativa. Esto es consonante con las lógicas actuales en las que la sociedad, las empresas, las corporaciones y los colectivos gestionan y producen conocimiento. Posiblemente esto se debe a la inteligencia colectiva en la que los seres humanos producimos colectivamente soluciones y en general conocimiento que es compartido en redes o nodos.

Un ejemplo de lo anterior es la figura 2, en la cual se visualizan centros de producción creados por los científicos a partir de estrategias de colaboración académica que favorecen la investigación, la innovación y el desarrollo. En cuanto al tamaño de los nodos, en este caso se conforma con base en el número de documentos publicados por cada autor. El grosor de las aristas indica el nivel de colaboración entre los autores, mientras que el color de los nodos se refiere a subcomunidades existentes dentro de la red.

Esta forma de representar la relación centro-periferia es propia de la era actual y supone que las comunidades cooperan con el fin de producir conocimiento. Esto se debe generar en el Ejército Nacional como materialización de la gestión investigativa y educativa centrada en el desarrollo de las competencias y capacidades.

Por eso los resultados de las investigaciones sobre las competencias investigativas ilustran de manera contundente la necesidad de seguir trabajando en la 


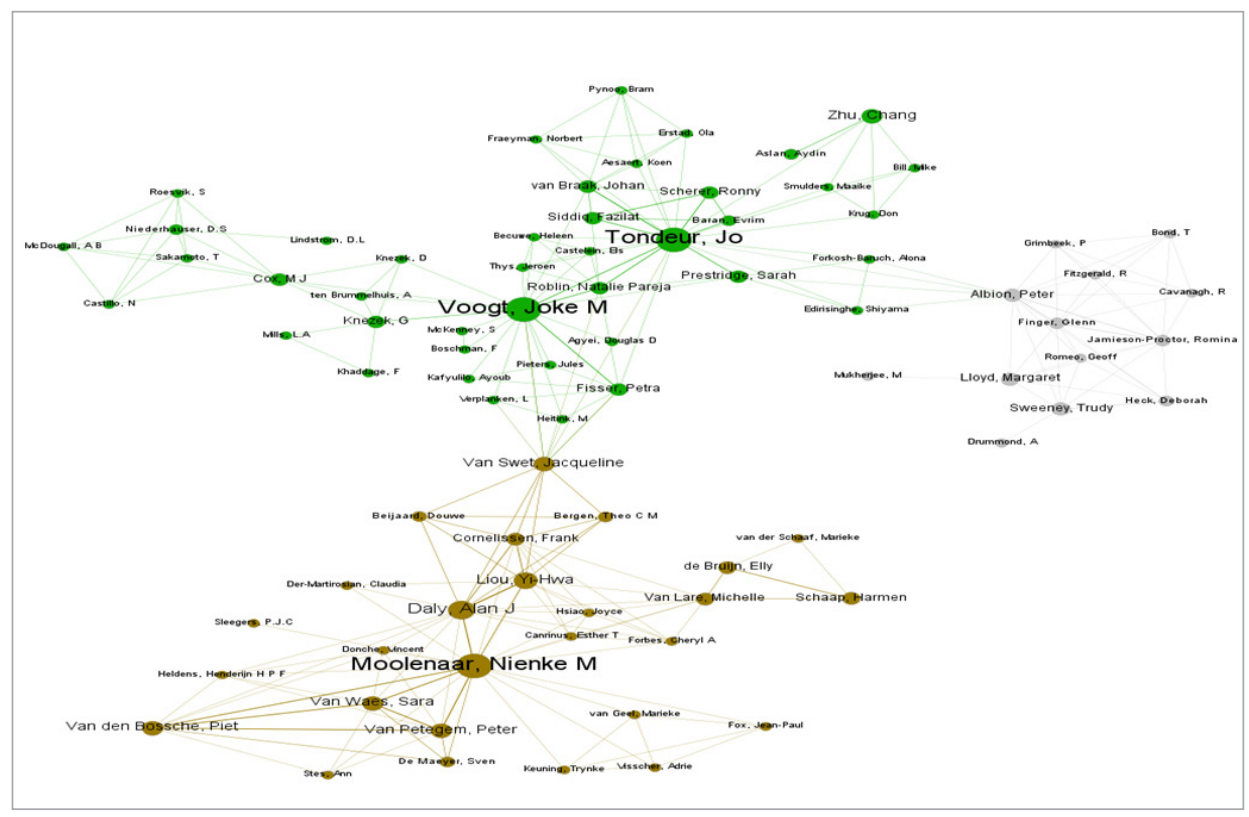

Figura 2. Red de autores con cuatro documentos publicados.

Fuente: Original de la autora.

formación y el desarrollo de comunidades de práctica centradas en la investigación, las cuales deben vincular a los estudiantes en experiencias reales de investigación (aprendizaje situado) con el propósito de desarrollar las competencias y habilidades básicas para la gestión, el desarrollo y la divulgación del conocimiento generado en las escuelas.

Aspectos como la motivación, los valores investigativos, el trabajo en equipo, la calidad de diseño investigativo, la calidad del producto referida al logro, las relaciones interpersonales, la comunicación y la colaboración son atributos importantes y categorías que permiten evaluar los procesos de investigación. Por esta razón la construcción de instrumentos de evaluación orientados a identificar, analizar y caracterizar las prácticas de formación en investigación y el desarrollo de la investigación científica en las escuelas es un gran aporte para asegurar la calidad educativa. Sobre todo si se considera que la sociedad actual supone un escenario de incertidumbre, dado que "por primera vez en la historia de la humanidad, la mayor parte de las competencias adquiridas por una persona al principio de su recorrido profesional estarán obsoletas al final de su carrera. Trabajar se convierte cada vez más en aprender; en transmitir saberes y producir conocimientos" (Lévy, 2007, p. 129). 
De acuerdo con lo anterior, es importante señalar el impacto de las afirmaciones de Lévy (2007) en el redimensionamiento curricular basado en competencias, pues como se analizó en las investigaciones, es una necesidad incluir aspectos no solo de orden disciplinar y procedimental, sino también aquellos socioafectivos relacionados con el liderazgo desde las perspectivas de género en el campo militar, pues esto es una condición necesaria a la luz de la defensa del desarrollo humano y la consolidación de sociedades democráticas e igualitarias. Esto implica transversalizar las diferentes dimensiones, contenidos temáticos sobre niveles, factores, estilos, tipos, atributos, aptitudes y destrezas de liderazgo, particularmente en los ciclos de formación. Hoy más que nunca la construcción de sociedades se basa en un respeto por la dignidad humana.

En este sentido, y considerando los principios y valores del Sistema Educativo de las Fuerzas Armadas, los resultados sobre la deserción de la población militar oficial millennial cobran un valor importante, toda vez que hace un llamado a los docentes, directivos y estudiantes sobre la importancia de analizar, comprender e identificar los factores asociados a la formación del contrato psicológico, pues esto puede mitigar los riesgos de deserción y aumentar la retención y el bienestar de los jóvenes que se incorporan a la institución.

Por tanto, los hallazgos de la investigación sugieren que el apoyo y seguimiento a las familias y el desarrollo de programas de bienestar a los militares es importante porque genera condiciones favorables para el cumplimiento de la misión. Asimismo, el sistema de educación y doctrina del Ejército Nacional debe articular y ampliar su oferta y cobertura a través de medios tecnológicos y alianzas con el mercado educativo, pues como se ha mostrado, las dinámicas de formación actual se caracterizan por su flexibilización y desterritorialización, lo cual ha generado mercados educativos globalizados.

Otro aspecto que incide en la permanencia de los millennial en la fuerza es el sistema de compensaciones actual, por eso debe ser revisado con el fin de incentivar al militar oficial millennial a cumplir su proyecto de vida. Finalmente, los investigadores sugieren fortalecer el sistema de valores y principios de la fuerza, utilizándolos como herramienta de identidad que permita retener a esta población.

A modo de cierre, se plantean los siguientes interrogantes para dejar posibles caminos para la investigación: ¿Cuáles son los saberes pedagógicos emergentes que fundamentan la enseñanza militar? ¿Cuáles son las prácticas de saber que orientan la praxis de los docentes, profesores e instructores militares? ¿Cuál es el impacto de la producción científica militar en la política pública? ¿Cómo se articulan las escuelas de formación militar con los centros de investigación civil? 


\section{Referencias}

Álvarez, A. (2018). El maestro en Colombia. En C. Noguera, A. Álvarez \& X. Herrera, Lecciones de historia de la pedagogía en Colombia. Escuela, maestro e infancia (pp. 87-157). Bogotá, D. C.: Magisterio.

Barragán, D. (2012). La práctica pedagógica, pensar más allá de las técnicas. En Barragán Gamboa \& Urbina (eds.), Prácticas pedagógicas (pp. 18 -38). Cúcuta: Universidad Francisco de Paula Santander.

Carr, W. (2002). Una teoría para la educación. Hacia una investigación educativa crítica (Trad. P. Manzano). Madrid: Morata.

Cortina, A. (2006). Universalizar la aristocracia: por una ética de las profesiones. Revista Santander, (1), 54-61. Recuperado de https://revistas.uis.edu.co/index.php/revistasantander/article/ view/2186/2547

Han, B. C. (2012). La sociedad del cansancio. Barcelona: Herder.

Lévy, P. (2007). Cibercultura: informe al Consejo de Europa (No. 16). Barcelona: Anthropos. 